\title{
¿Profesores de instrumento o profesores como instrumento? De lo transferencial a lo transformativo en los enfoques de la pedagogía uno-a-uno

\author{
Teachers of instruments, or teachers as instruments? From
} transfer to transformative approaches to one-to-one pedagogy
}

\author{
por \\ Gemma Carey \\ Queensland Conservatorium, Griffith University, Australia \\ g.carey@griffith.edu.au \\ Catherine Grant \\ Queensland Conservatorium, Griffith University, Australia \\ catherine.grant@griffith.edu.au
}

Traducción

Mauricio Valdebenito Cifuentes

Facultad de Artes, Universidad de Chile, Chile

mvaldebe@uchile.cl

La investigación en pedagogía en el contexto de la formación musical vocal e instrumental es, por su naturaleza, ilimitada, en la medida que los docentes buscan constantemente mejorar el apoyo y desarrollo en el aprendizaje de sus estudiantes. De acuerdo con la bibliografía y los datos cualitativos y cuantitativos de un proyecto en curso en una institución de enseñanza musical terciaria en Australia, este trabajo cuestiona algunos de los enfoques recurrentes en materia de enseñanza individual, resultando en un replanteamiento profundo del rol del profesor de canto e instrumento. Los autores contrastan una pedagogía transferencial basada en una didáctica tradicional centrada en el maestro con otra centrada en el estudiante y con un enfoque del aprendizaje enriquecido por el contexto. Esta pedagogía transformativa se caracteriza por un gran compromiso de los estudiantes con su formación, una mayor riqueza conceptual y una mejora general de los resultados de aprendizaje. Los alcances de este estudio consideran: el compromiso por parte de los profesores con la reflexión crítica de sus enfoques pedagógicos en el contexto uno-a-uno ${ }^{1}$; que lo estudiantes sean concientes de los beneficios que se producen cuando ellos mismos se apropian y responsabilizan de su propio aprendizaje; y para las instituciones, explorar ampliamente el panorama y naturaleza de la formación vocal e instrumental, y el apoyo colaborativo y reflexivo de las estrategias de aprendizaje por medio de y entre profesores y estudiantes.

Palabras clave: Enseñanza instrumental; pedagogía uno-a-uno; educación musical terciaria; pedagogía transformativa; enseñanza vocal.

1 En adelante, la expresión "uno-a-uno" es igual a "clase individual”, es decir, cuando una clase se realiza con la participación de un docente y un estudiante.

Revista Musical Chilena, Año LXXV, julio-diciembre, 2021, No 236, pp. 175-183 Fecha de recepción: 14-09-2020. Fecha de aceptación: 26-01-2021 
Research into pedagogy in the context of instrumental and vocal tuition is, by its nature, limitless, as teachers constantly seek improved ways to support and develop their students' learning. Drawing on the literature as well as qualitative and quantitative data from a project underway at one Australian tertiary music institution, this paper challenges some common existing approaches to one-to-one tuition, prompting a rethink of the fundamental role of the instrumental and vocal teacher. The authors contrast the traditional, didactic, teacher-oriented transfer pedagogy with a student-oriented, explorative, context-rich approach to learning. This transformative pedagogy is characterized by greater student engagement in learning, stronger conceptual understanding, and improved learning outcomes overall. Implications of the study include the imperative for teachers to engage in ongoing critical reflection of their pedagogical approach in the one-to-one context; for students to be made aware of the benefits that eventuate when they take ownership of and responsibility for their learning; and for institutions to explore broadening the scope and nature of instrumental and vocal tuition, and to support collaborative and reflective learning strategies among and between both students and teachers.

Keywords: instrumental teaching, one-to-one pedagogy, tertiary music education, transformative pedagogy, vocal teaching.

\section{APROXIMACIONES A LA ENSEÑANZA INDIVIDUAL ${ }^{2}$}

La enseñanza en la modalidad de clase individual ha sido la columna vertebral de la educación musical durante los dos últimos siglos, y es el modelo que, hasta hoy, se mantiene como el más familiar para la mayoría de los músicos instrumentistas y cantantes, sean estos estudiantes o profesionales. Si bien para muchos estudiantes el primer contacto con la educación musical se produce en la escuela, prácticamente todos los músicos profesionales optan -en algún momento de sus vidas- por las clases individuales como su principal método de formación musical (Carey 2008). Una investigación realizada en dos instituciones de educación musical de nivel terciario, durante el 2008 y 2009, señala que una gran mayoría de sus estudiantes tuvo previamente un mínimo de cincuenta lecciones individuales (Lebler, Burt-Perkins y Carey 2009). Este mismo estudio informa, además, que estas clases de música son a menudo las únicas experiencias de aprendizaje individual que los estudiantes tienen en cualquier materia a lo largo de toda su educación formal. Solo por esta razón, los profesores que realizan clases individuales tienen una influencia potencial enorme respecto de muchos aspectos relacionados con el aprendizaje y desarrollo de sus estudiantes, y no es de extrañar entonces que los profesores se conviertan en personas muy importantes en sus vidas.

Debido al importante papel que cumplen los profesores de música en el crecimiento y desarrollo de los estudiantes, sería razonable suponer que existe un sistema eficaz de seguimiento y regulación de su formación, calificaciones y acreditación. Sin embargo, este no es el caso. No existe un sistema de acreditación a pesar de la insistencia que investigaciones recientes señalan en cuanto a la necesidad de contar con mejores sistemas de formación para profesores de voz e instrumento (e.g. Carey y Harrison 2007; Bennett 2008; Carey 2008; Creech et al. 2008; Gaunt 2009). Incluso en la elite de instituciones musicales como los conservatorios, los profesores de voz e instrumento son, en su mayoría, contratados sobre la base de sus competencias como intérpretes más que como profesores, a pesar de que el conjunto de habilidades requerido para cada uno de estos roles es muy diferente. En muchos casos, los profesores de conservatorio aprenden a enseñar "en el trabajo mismo", aumentando las probabilidades de que, como es de esperar, adopten por defecto los métodos y enfoques pedagógicos con los que ellos mismos fueron formados. Así, de un modo circular, la naturaleza de la educación del conservatorio no solo se mantiene "prolongadamente sin investigar", sino que además permanece "crucial y sostenidamente sin contrapeso" (Perkins 2013).

Se trata de una situación riesgosa. Estudios recientes en pedagogía uno-a-uno en conservatorios indican que, mientras el modelo de clase individual puede significar un tipo de seducción inicial por parte de los estudiantes (por ejemplo, en términos de contar con una atención y guía personalizada

2 Carey, G. y Grant, C. (2014). Teachers of instruments, or teachers as instruments? From transfer to transformative approaches to one-to-one pedagogy. Relevance and reform in the education of professional musicians, Glen Carruthers (editor), pp. 42-54. Proceedings of the 20th International Seminar of the ISME Commission on the Education of the Professional Musician (CEPROM), 15-18 July 2014, Belo Horizonte, Brazil. 
por parte del profesor), a largo plazo esto puede tener consecuencias negativas (Persson 1994; Burwell 2006; Carey y Grant 2014). Estos peligros consideran la creación de una cultura de dependencia del profesor; el riesgo concomitante de que el estudiante se convierta en un sujeto pasivo, incapaz de trabajar con autonomía, y por tanto, desilusionado con su propia capacidad de aprendizaje; la incapacidad del estudiante para adaptar su aprendizaje a diversos contextos musicales fuera de los límites absorbentes de su propio ambiente de estudio; un impacto negativo en su desarrollo musical (y otros atributos personales tales como confianza en sí mismo e iniciativa) en ciertos tipos de relación profesorestudiante; y en particular para estudiantes de la educación terciaria, la incapacidad para desarrollar, en un sentido amplio, las habilidades necesarias para forjar una carrera profesional exitosa como músico (Jorgensen 2000; Mills 2002; Burwell 2005; Carey 2008; Gaunt 2008, 2010; Gaunt, Creeech, Long y Hallam 2012). Adicionalmente, cualquier otro efecto adverso se agrava por el hecho de que comúnmente, estudiantes con experiencias de aprendizaje instrumental o vocal en la modalidad individual están llamados a convertirse en la siguiente generación de educadores, y al recurrir a sus propias experiencias de enseñanza en la construcción de sus enfoques pedagógicos el ciclo se perpetúa.

Aunque algunos de estos riesgos pueden manifestarse en cualquier contexto de clase individual, algunos estudios sugieren que estos podrían ser aún más pronunciados en situaciones donde el profesor adopta un enfoque de "transferencia" hacia la enseñanza y el aprendizaje (Carey et al. 2013b). Una pedagogía transferencial es didáctica por naturaleza, comúnmente basada en la instrucción, el modelo, la demostración, la imitación al profesor, la pasividad del estudiante y un aprendizaje descontextualizado. Además, está caracterizada por una aproximación pedagógica predeterminada, con nociones de excelencia definidas y determinadas, y donde los logros del aprendizaje tienden a la focalización más que a la expansión. La evaluación, entendida como un punto final, orienta el aprendizaje, y el desarrollo de habilidades musicales y técnicas constituye un elemento central (Carey et al. 2013b; Carey y Grant 2014). Si bien una aproximación de estilo transferencial para la enseñanza uno-a-uno ha demostrado resultados de aprendizaje, especialmente en términos del desarrollo de habilidades musicales y técnicas, los riesgos mencionados anteriormente plantean las siguientes cuestiones ¿Es el enfoque convencional uno-a-uno en la instrucción vocal e instrumental -en los hechos- un impedimento para el aprendizaje de los estudiantes en el siglo XXI? Y si es así ¿Qué otros métodos pedagógicos podrían emplear los profesores para optimizar el aprendizaje de los estudiantes?

\section{"REPENSAR" LA MODALIDAD UNO-A-UNO}

En un nivel general, mucha de la investigación actual en pedagogía efectiva destaca la necesidad de un cambio en el enfoque que vaya más lejos de aquel centrado únicamente en la autoridad del maestro. Los estudios en el contexto de la educación superior han evidenciado que los estudiantes están desilusionados con los enfoques centrados en el maestro (Barnes y Tynan 2007), y se distancian cada vez más de modelos de enseñanza y aprendizaje que consideran obsoletos (Barnett y Coate 2005). Las teorías educacionales contemporáneas han pasado de considerar a los estudiantes como receptores pasivos de conocimiento, a un modelo inclusivo donde los estudiantes son participantes activos que controlan su propio aprendizaje, incluida la posibilidad de aportar información del contenido y los procesos.

Este cambio de paradigma tiene sus raíces en teorías pedagógicas aún más antiguas, como el pensamiento constructivista de Piaget (1970) y Vygostsky (1978). Los estudiantes exploran, experimentan, cuestionan y reflexionan acerca de problemas del mundo real, actuando como agentes activos en su propio aprendizaje, aprendiendo cómo aprender, y desarrollando habilidades transferibles en el proceso. El rol del profesor es, esencialmente, proveer a los estudiantes la guía necesaria, así como las herramientas y recursos para gestionar su propio aprendizaje. En su mayoría, las investigaciones educativas de vanguardia respaldan firmemente estas filosofías, señalando que los estudiantes adquieren una comprensión conceptual más sólida de los contenidos, un mayor involucramiento en su aprendizaje y el logro de mejores resultados cuando ellos mismos encaminan y direccionan su propio desarrollo, donde se enfatiza el proceso por sobre el contenido, y donde los resultados transformacionales se valoran más que la sola asimilación de información en un nivel superficial (Dirkx, Mezirow y Cranton 2006; Lysaker y Furuness 2011; King 2005; McGonigal 2005).

En el contexto de este estudio acerca de la modalidad de enseñanza individual en música, el enfoque se traduce en que el papel del maestro es crear una situación en la que los estudiantes aprenden a enseñarse a sí mismos. Y aunque el maestro puede proporcionarle al estudiante instrucciones claras, el tiempo dedicado a explicar, demostrar y exigir que los estudiantes imiten es acotado, lo que 
da más tiempo para que experimenten y aprendan de sus propios logros y errores. De esta forma, el estudiante se ubica en el centro del aprendizaje. Por su parte, el maestro no es solo un profesor de instrumento sino un "instrumento" para el aprendizaje.

Este enfoque hacia una enseñanza vocal e instrumental puede denominarse pedagogía transformativa (Carey et al. 2013b; citado en Boyd y Myers 1988; ver también Mezirow 1997, 2000; Mezirow 2000; McGonigal 2005; Taylor 1998, 2007). Los profesores que trabajan en el estilo transformativo están orientados hacia el aprendizaje más que a la evaluación, su principal objetivo es la excelencia "expansiva" más que la excelencia "definida" que caracteriza a profesores de estilo transferencial. En el estilo transformativo el contenido a enseñar se articula y contextualiza, ayudando al estudiante a entender su propio aprendizaje, por ejemplo, situándolo dentro del amplio contexto de su carrera y su vida. Adoptan un enfoque abierto, colaborativo y exploratorio en sus estudios, alentando a sus estudiantes a tomar posesión efectiva de su aprendizaje. En sus opciones pedagógicas permanecen ágiles y flexibles, respondiendo a las necesidades individuales de los estudiantes. Por último, los profesores de música que adoptan una pedagogía transformativa son capaces de "promover tanto el rendimiento como los resultados de aprendizaje en sus estudiantes, aunque su objetivo principal pone énfasis precisamente en el aprendizaje (esto es, en el aumento de la capacidad mediante nuevos conocimientos o desarrollo de habilidades)" en lugar del rendimiento (Carey et al. 2013b).

Para los estudiantes, este enfoque en la enseñanza y el aprendizaje tiene beneficios inmediatos y tangibles. El pensamiento independiente desarrollado por medio del aprendizaje transformador ayuda a mantener el interés en aprender y estimula la motivación. Tanto la experiencia como los conocimientos previos de los estudiantes se ubican en la vanguardia de su aprendizaje, creando confianza y autonomía. De igual modo, los estudiantes son capaces de transferir su aprendizaje a otros contextos. Más importante aún, la pedagogía transformativa contribuye al logro de lo que constituye el objetivo principal de cualquier proceso educativo: desarrollar en el estudiante la capacidad de autocontrol, autocrítica y autogestión para continuar aprendiendo autónomamente en el futuro.

\section{UN CASO DE ESTUDIO INSTITUCIONAL}

$\mathrm{Al}$ interrogar las características y procesos de la pedagogía vocal e instrumental uno-a-uno, un proyecto de investigación realizado en la institución donde trabajan las autoras de este artículo en Australia subraya algunos de los problemas relacionados con la modalidad uno-a-uno y el papel del maestro. Investigadores y profesores trabajaron asociativamente en el diseño y ejecución del proyecto, que incluyó la realización de focus groups, entrevistas a profesores, y un extenso análisis videográfico de varias clases individuales (uno-a-uno). Tanto la metodología como los resultados preliminares se describen en profundidad en publicaciones anteriores y separadas (Carey, Grant, McWilliam y Taylor 2013, Carey, Bridgstock, Taylor, McWilliam y Grant 2013). Los seis maestros involucrados en la videografía mostraron características tanto de la pedagogía transformativa como transferencial, pero en general, tendieron hacia un estilo de transferencia; solo un pequeño grupo de profesores adoptó características asociadas mayoritariamente a la pedagogía transformativa. Además, aunque los profesores de estilo transferencial alcanzaron en sus estudiantes resultados tanto performativos como de aprendizaje, el énfasis en aspectos performativos fue mucho mayor. Y aunque todos los profesores declararon una intención para promover el aprendizaje independiente, para aquellos profesores de estilo predominantemente transferencial esto no se tradujo en prácticas pedagógicas concretas.

El mapeo y contraste entre las prácticas de docentes y estudiantes en la modalidad uno-a-uno confirmó el valor de la enseñanza y el aprendizaje transformador. Todos los profesores sintieron que la capacidad de fomentar en sus estudiantes habilidades de aprendizaje independiente era una prioridad. "No quiero producir réplicas de mí misma -ni mucho menos-. Quiero que los estudiantes conserven su propia voz... siempre intento promover la independencia musical" (Profesor 3 , mujer). Otra profesora estuvo de acuerdo en que su rol era alentar a los estudiantes a tomar el control de su propio aprendizaje: "Mi principal objetivo es que (los estudiantes) sean autosuficientes" (Profesor 4, mujer).

Los estudiantes también hicieron ver su deseo y necesidad de ser responsables de su propio aprendizaje: "Se trata de una calle con doble sentido, y no de un solo sentido" (Estudiante 1, hombre). Otro reconoció que: "Un profesor solo puede dar lo mejor de sí mismo en una situación uno-a-uno. Ellos solo pueden orientarte en una sola dirección antes de que tú puedas tomar el control" (Estudiante 4, mujer). Sin embargo, varios estudiantes expresaron su preocupación acerca de los riegos del 
aprendizaje autónomo. Una estudiante reflexionó así por su experiencia en el primer año de estudios en el conservatorio:

Al entrar, la profesora me preguntó con qué técnica y con cuáles estudios quería comenzar. Y yo simplemente estaba en primer año: Y necesitaba que me dieran algo que pudiera entender... y solo sentía... como si hubiera sido arrojada al fondo (Estudiante 1, mujer).

Algunos profesores demostraron tener conciencia que para algunos estudiantes la independencia y el dominio de su propio aprendizaje (pedagogía transformativa) necesitan incrementarse gradualmente a medida que los estudiantes desarrollan estas habilidades. "En el primer año mis ejemplos serán más, luego en el segundo y tercer año serán probablemente cada vez menos. Hacia el cuarto año, diría que es esperable que las lecciones sean... mucho más dirigidas por el propio estudiante antes que el profesor" (Profesor 3, mujer).

Además de trabajar horizontalmente con sus estudiantes, los profesores también constataron el valor de trabajar de manera más colaborativa entre ellos, aprovechando la experiencia y habilidades de sus colegas. De esta manera, el profesor se convierte en un participante en el proceso de aprendizaje. Tres de los seis docentes que participaron del estudio indicaron que, de alguna manera, habían adoptado este enfoque. Otro invitó a más profesionales a su clase, en el entendido que esto "no solo aporta una mayor experiencia a la sesión, de la cual me beneficio yo tanto como el estudiante, sino que también es un reflejo de la vida profesional, donde aprendes de una variedad infinita de fuentes" (Profesor 7, mujer). Otro profesor adoptó un enfoque de enseñanza en equipo, con estudiantes rotando entre diferentes maestros en sus primeros dos años de estudio, y seleccionando una combinación de profesores para trabajar en el tercer año (Profesor 4, mujer). Algunos profesores reconocieron el valor de realizar lecciones individuales en combinación con varios otros modelos o formatos, tales como: el aprendizaje entre pares mediante un trabajo en conjunto, el trabajo en ensambles pequeños, tocando entre ellos y con estudiantes mayores que "puedan guiar o cautelar" a los más jóvenes, musicalmente hablando (Profesor 2, hombre).

\section{CONCLUSIONES}

Aunque muchos estudiantes son formados en conservatorios con la esperanza de alcanzar un desempeño profesional como músicos de élite, un buen número forjará una carrera que involucrará, en algún grado, la enseñanza (Bennett 2008). Y para aquellos a quienes se enseña se debe considerar la opción de que puedan estar más motivados por el deseo de disfrutar de su aprendizaje, desarrollar su amor y aprecio por la música, así como las habilidades para apoyar otras actividades musicales recreativas a lo largo de sus vidas, antes que por una búsqueda de la excelencia. Sin embargo, es muy probable que aquellos estudiantes-maestros con experiencia en el estilo transferencial de la modalidad uno-a-uno serán proclives a adoptar el mismo estilo de transferencia en su propia enseñanza, una probabilidad irónicamente amplificada por el hecho de que estos individuos pueden replicar estas prácticas institucionalizadas precisamente porque no tuvieron la oportunidad de "aprender a aprender" en su propia educación. Si este es el caso -y se requiere aún más investigación para confirmarlo- los estudiantes de conservatorio con experiencias en aprendizajes de transferencia parecen tener menos probabilidades de éxito en el futuro para ayudar a sus propios estudiantes a alcanzar objetivos de aprendizaje transformadores. Por otra parte, aquellos con experiencias transformadoras en la modalidad uno-a-uno saben cómo estimular, apoyar y guiar el aprendizaje. Cuando los enfoques transformadores hacia la pedagogía uno-a-uno sean una práctica regular en nuestros conservatorios el ciclo se romperá.

Para los profesores, la decisión de pasar de un enfoque predominantemente transferencial a otro transformador en una pedagogía individual, tiene profundas implicancias. Con una función profundamente modificada desde una posición de instructor autorizado hacia una de colaborador y facilitador, los profesores tendrán que renunciar a su posición de expertos y, al mismo tiempo, aceptar una mayor diversidad de estilos de aprendizaje, estructuras y resultados de los que pudieran estar habituados mediante el enfoque de transferencia, más orientado a la evaluación. En consecuencia, los profesores podrían necesitar desarrollar nuevas habilidades para monitorear, facilitar y guiar a los estudiantes. Para que la transformación de la enseñanza de la música en la modalidad uno-a-uno sea exitosa, los docentes deben cuestionarse permanentemente acerca de lo que están aprendiendo sus estudiantes, es decir, si creen que lo que están enseñando es, en los hechos, aquello que está siendo 
entregado (aprendido). En este sentido, sistemas bien consolidados de una práctica reflexiva y crítica serán esenciales para aquellos profesores que opten por cambiar su enfoque (Mezirow 1991; Brookfield 1995; Cranton y Carusetta 2004; Kreber 2004; Lysaker y Furuness 2011).

Los estudiantes, por su parte, requerirán estar abiertos a nuevas experiencias en el contexto de la modalidad uno-a-uno. Para que un enfoque transformativo en la enseñanza resulte exitoso, los estudiantes deben asumir una enorme responsabilidad en su proceso de aprendizaje, por ejemplo, siendo más proactivos en sus propios cuestionamientos y objetivos de aprendizaje. Asimismo, los estudiantes podrían ser reticentes a adoptar enfoques pedagógicos desconocidos, especialmente en el caso de quienes prefieren la seguridad de un enfoque pasivo, prescriptivo u otro orientado hacia un estilo de aprendizaje centrado en la resolución de tareas (cf. Murphy 2009; Minhas, Ghosh y Swanzy 2012). Por tanto, la evaluación de estudiantes con una enseñanza transformadora -especialmente en contextos donde se ha realizado un cambio hacia este tipo de enfoques transformativos- requerirá considerar la posibilidad de un contexto en que, como muchos de nosotros, también los estudiantes sean resistentes al cambio.

En el caso de las instituciones, dos son las principales implicancias de estas conclusiones. En primer lugar, la enseñanza de la música en la modalidad uno-a-uno resulta más valiosa cuando adopta características transformativas. Sobre la base de la evidencia disponible, esta afirmación solo es aplicable con relación a resultados de aprendizaje genéricos y transmisibles (aquello que en el sector de la educación superior se conoce como "perfil de egreso" [graduate atributes]), y no a resultados específicos de la disciplina tales como el desarrollo técnico y musical. Por tanto, se trata de un área abierta para futuras investigaciones. La segunda implicancia es inferencial: esto es, que a pesar de los muchos beneficios en la modalidad uno-a-uno, proporcionar acceso a una combinación de diferentes pedagogías podría incrementar el aprendizaje de los estudiantes contribuyendo a un entorno de aprendizaje transformativo. Las actividades de aprendizaje colaborativo (van der Linden et al. 2000; Gaun y Westerlund 2013) consideran a los estudiantes como parte de una práctica comunitaria que puede contrarrestar la intensidad, a menudo interna, de la modalidad uno-a-uno. Gaunt, Creech, Long y Hallam (2012) descubrieron que los estudiantes de conservatorio con más de un maestro eran más responsables de su propio progreso que aquellos en una relación de aprendizaje exclusiva. Renshaw (2009) describe experiencias de profesores y orientadores efectivos en el contexto musical; y varios estudios conceptualizan la modalidad uno-a-uno como una "colaboración creativa" y destacan los beneficios de llevarla a la práctica (Presland 2005; Barrett y Gromko 2007; Gaunt, Creech, Long y Hallam 2012; Burwell 2013). Es importante señalar que los enfoques colaborativos para el aprendizaje pueden beneficiar no solo a estudiantes de canto o instrumento, sino que también a los propios maestros, quienes pueden beneficiarse de la oportunidad de reflexionar, participar y mejorar, de forma colaborativa y participativa, acerca de sus prácticas docentes (Conway 2006; Haack 2006; Blair 2009; Haack y Smith 2009).

Para el caso de profesores de instrumento y canto en el siglo XXI el desafío es continuar mejorando los enfoques pedagógicos y los resulados de aprendizaje de los estudiantes. Solo investigaciones recientes han comenzado a exponer en profundidad las características comunes de la enseñanza musical de clases individuales (uno-a-uno) y los supuestos que la sustentan, debido, al menos en parte, a las dificultades involucradas en el acceso al espacio privado e íntimo de esas clases (Carey 2008). Debido a la creciente atención académica sobre la naturaleza, beneficios y desafíos de este enfoque pedagógico, las posibilidades para los profesores se amplían, no solo para recurrir a tradiciones de enseñanza del pasado -unas veces excelentes y otras no tanto-, sino que también para aprender, criticar, explorar y eventualmente, llegado el caso, adoptar enfoques educativos innovadores que permitan mejorar las habilidades y capacidades de los estudiantes. En este sentido, la enseñanza y el aprendizaje continuarán siendo relevantes aquí y ahora. Por tanto, el argumento presentado aquí no es que se abandonen por completo los enfoques más convencionales basados en la transferencia en la modalidad uno-a-uno, sino que tanto los profesores como las instituciones puedan permanecer profundamente comprometidos en la práctica reflexiva y abiertos a incorporar prácticas pedagógicas transformadoras que mejoren las capacidades de nuestros estudiantes para aprender y crecer como músicos, como futuros maestros, y también como personas. 


\section{BIBLIOGRAFÍA}

Barnes, Cameron y Belinda Tynan

2007 "The adventures of Miranda in the brave new world: Learning in a Web 2.0 millennium", Research in Learning Technology, XV/3, pp. 189-200. doi: 10.1080/09687760701673568

Barnett, Ronald y Kelly CoAte

2005 Engaging the curriculum in higher education. Buckingham, UK: SRHE \& Open University Press.

Barrett, Margaret S. y Joyce Eastlund Gromko

2007 "Provoking the muse: A case study of teaching and learning in music composition", Psychology of Music, XXXV/2, pp. 213-230. doi: 10.1177/0305735607070305.

BennetT, DAWN

2008 "Portfolio careers and the conservatoire", Inside Outside Downside Up: Conservatoire Training and Musicians' Work. Dawn Bennett y Michael Hannan (editores). Perth: Black Swan Press, pp. 61-72.

Blair, Deborah V.

2009 "Mentoring novice teachers: Developing a community of practice", Research Studies in Music Education, XXX/2, pp. 99-117. doi: 10.1177/1321103X08097502.

Boyd, Robert D. y J. Gordon Myers

1988 “Transformative education", International Journal of Lifelong Education, 7, pp. 261-284. doi: $10.1080 / 0260137880070403$.

Brookfield, STEPHeN D.

1995 Becoming a critically reflective teacher. Nueva York: John Wiley.

BurWELL, Kim

2005 "A degree of independence: Teachers' approaches to instrumental tuition in a university college", British Journal of Music Education, XXII/3, pp. 199-215. doi: 10.1017/ S0265051705006601.

2006 "On musicians and singers: An investigation of different approaches taken by vocal and instrumental teachers in higher education”, Music Education Research, VIII/3, pp. 331-347. doi: 10.1080/14613800600957479.

2013 "Apprenticeship in music: A contextual study for instrumental teaching and learning", International Journal of Music Education, XXXI/3, pp. 276-291. doi: 10.1177/0255761411434501.

CAREY, GEMMA

2008 New understanding of 'relevant' keyboard pedagogy in tertiary institutions. Saarbrucken: Verlag Dr. Muller.

2010 "Too much performing, too little learning? Reflections on pedagogical practices within the Conservatoire (ponencia)", 18th International Seminar of the Commission for the Education of the Professional Musician (CEPROM), International Society for Music Education (ISME), Nedlands, Western Australia.

Carey, Gemma y Catherine Grant

2014 "Teacher and student perspectives on one-to-one pedagogy: Practices and possibilities", British Journal of Music Education XXXII/1, pp. 5-22. doi: 10.1017/S0265051714000084.

Carey, Gemma y Scott Harrison

2007 "The practice of pedagogy (ponencia)", National Conference of NACTMUS, Brisbane, 29 de junio de 2007. Disponible en: https://www.researchgate.net/profile/Scott_Harrison3/ publication/29466338_The_Practice_of_Pedagogy/links/00b49517842ad322c6000000.pdf [acceso: 14 septiembre de 2013]

Carey, Gemma, Catherine Grant, Erica McWilliam y Peter Taylor.

2013 "One-to-one pedagogy: Developing a protocol for illuminating the nature of teaching in the conservatoire", International Journal of Music Education, XXXI/2, pp. 148-159. doi: $10.1177 / 0255761413483077$. 
Carey, Gemma, Ruth Bridgstock, Peter Taylor, Erica McWilliam y Catherine Grant.

2013 "Characterising one-to-one conservatoire teaching: Some implications of a quantitative analysis”, Music Education Research, XV/3, pp. 357-368. doi: 10.1080/14613808.2013.824954.

Conway, Colleen M.

2006 "Navigating through induction: How a mentor can help", Music Educators Journal, XCII/5, pp. 56-60. doi: 10.2307/3878504.

Cranton, Patricia y Elleen Carusetta

2004 "Developing authenticity as a transformative process", Journal of Transformative Education, II/4, pp. 276-293. doi: 10.1177/1541344604267898.

Creech, Andrea et al.

2008 "From music student to professional: The process of transition", British Journal of Music Education, XXV/3, pp. 315-331.

Dirkx, John M., Jack Mezirow y Patricia Cranton

2006 "Musings and reflections on the meaning, context, and process of transformative learning: A dialogue between John M. Dirkx and Jack Mezirow", Journal of Transformative Education, IV/2, pp. 123-139. doi: 10.1177/1541344606287503.

Gaunt, Helena

2008 "One-to-one tuition in a conservatoire: The perceptions of instrumental and vocal teachers", Psychology of Music, XXXVI/2, pp. 215-245. doi: 10.1177/0305735607080827.

2010 "One-to-one tuition in a conservatoire: The perceptions of instrumental and vocal students", Psychology of Music, XXXVIII/2, pp. 178-208. doi: 10.1177/0305735609339467.

Gaunt, Helena y Heidi Westerlund

2013 Collaborative Learning in Higher Music Education. Surrey, UK: Ashgate.

Gaunt, Helena, Andrea Creech, Marion Long y Susan Hallam

2012 "Supporting conservatoire students towards professional integration: One-to-one tuition and the potential of mentoring", Music Education Research, XIV/1, pp. 25-43. doi: $10.1080 / 14613808.2012 .657166$.

Haack, Paul A.

2006 "Mentoring and professional development programs: Possibilities and pitfalls", Music Educators Journal, XCII/4, pp. 60-64. doi: 10.2307/3401114.

HaAck, Paul A. y Michael V. Smith

2009 "Mentoring new music teachers", Music Educators Journal, LXXXVII/3, pp. 23-27. doi: $10.2307 / 3399659$.

JORGENSEN, HARALD

2000 "Student learning in higher instrumental education: Who is responsible?", British Journal of Music Education, XVII/1, pp. 67-77. doi: 10.1017/S0265051700000164.

King, KathleEN P.

2005 Bringing transformative learning to life. Malabar, FL: Krieger.

Kreber, Carolin

2004 "An analysis of two models of reflection and implications for educational development", International Journal for Academic Development, IX/1, pp. 29-49. doi: $10.1080 / 1360144042000296044$.

Lebler, Don, Rosie Burt-Perkins y Gemma Carey

$2009 \quad$ "What the students bring: Examining the attributes of commencing conservatoire students", International Journal of Music Education, XXVII/3, pp. 232-249. doi: $10.1177 / 0255761409335950$.

LySAKer, JUdith y SHELly Furuness

2011 "Space for transformation: Relational, dialogic pedagogy", Journal of Transformative Education, IX/3, pp. 183-187. doi: 10.1177/1541344612439939. 
McGonigal, Kelly

2005 "Teaching for transformation: From learning theory to teaching strategies", Speaking of Teaching, XIV/2, pp. 1-4.

Mezirow, JaCK

1991 Transformative dimensions of adult learning. San Francisco: Jossey-Bass.

1997 "Transformative learning: Theory to practice”, New Directions for Adult and Continuing Education, 74, pp. 5-12.

2000 "Learning to think like an adult", Learning as transformation: Critical perspectives on a theory in progress. Jack Mezirow (editor). San Francisco: Jossey-Bass, pp. 3-33.

Mills, Janet

2002 "Conservatoire students' perceptions of the characteristics of effective instrumental and vocal tuition”, Bulletin of the Council for Research in Music Education, 153/154, pp. 78-82.

Minhas, Paras Singh, Arundhati Ghosh y Leah Swanzy

2012 "The effects of passive and active learning on student preference and performance in an undergraduate basic science course", Anatomical Sciences Education, V/4, pp. 200-207. doi: 10.1002 /ase.1274.

Murphy, NoEla

2009 "Research supervision: Matches and mismatches", International Journal of Electrical Engineering Education, XLVI/3, pp. 295-306. doi: 10.7227/IJEEE.46.3.7.

Perkins, Rosie

2013 "Learning cultures and the conservatoire: An ethnographically-informed case study", Music Education Research, XV/2, pp. 196-213. doi: 10.1080/14613808.2012.759551.

Persson, Roland S.

1994 "Concert musicians as teachers: On good intentions falling short", European Journal for High Ability, 5, pp. 79-91. doi: 10.1080/0937445940050108.

Piaget, JeAN

1970 Genetic epistemology. Nueva York: Columbia University Press.

Presland, Carole

2005 "Conservatoire student and instrumental professor: The student perspective on a complex relationship”, British Journal of Music Education, XXII/3, pp. 237-248. doi: 10.1017/ S0265051705006558.

Renshaw, Peter

2009 Lifelong learning for musicians: The place of mentoring. Hanzehogeschool Groningen.

TAYLOR, EDWARD W.

1998 The theory and practice of transformative learning: A critical review. Columbus, OH: ERIC Clearinghouse on Adult, Career, and Vocational Education.

2007 "An update of transformative learning theory: A critical review of the empirical research (1999-2005)", International Journal of Lifelong Education, XXVI/2, pp. 173-191. doi: $10.1080 / 02601370701219475$.

Van der Linden, Jos, Gisjbert Erkens, Henk Schmidt y Peter Renshaw

$2000 \quad$ "Collaborative learning”, New Learning. Robert-Jan Simons, Jos van der Linden y Tom Duffy (editores). Dordrecht: Kluwer Academic, pp. 37-54.

Vygotsky, LeV S.

1978 Mind in Society. Cambridge, MA: Harvard University. 